\title{
Towards practical quantum computers: transmon qubit with a lifetime approaching 0.5 milliseconds
}

Chenlu Wang ${ }^{1}$, Xuegang $\mathrm{Li}^{1}$, Huikai Xu ${ }^{1}$, Zhiyuan $\mathrm{Li}^{1}$, Junhua Wang ${ }^{1}$, Zhen Yang ${ }^{1}$, Zhenyu Mi ${ }^{1}$, Xuehui Liang ${ }^{1}$, Tang Su${ }^{1}$, Chuhong Yang ${ }^{1}$, Guangyue Wang ${ }^{1}$, Wenyan Wang ${ }^{1}$, Yongchao $\mathrm{Li}^{1}$, Mo Chen ${ }^{1}$, Chengyao Li ${ }^{1}$, Kehuan Linghu ${ }^{1}$, Jiaxiu Han ${ }^{1}$, Yingshan Zhang ${ }^{1}$, Yulong Feng ${ }^{1}$, Yu Song ${ }^{1}$, Teng Ma ${ }^{1}$, Jingning Zhang ${ }^{1}{ }^{1}$, Ruixia Wang ${ }^{1}$, Peng Zhao ${ }^{1}$, Weiyang Liu (iD) Guangming Xue ${ }^{1 凶}$, Yirong $\operatorname{Jin}^{1 凶}$ and Haifeng $\mathrm{Yu}^{1 凶}$

Here we report a breakthrough in the fabrication of a long lifetime transmon qubit. We use tantalum films as the base superconductor. By using a dry etching process, we obtained transmon qubits with a best $T_{1}$ lifetime of $503 \mu \mathrm{s}$. As a comparison, we also fabricated transmon qubits with other popular materials, including niobium and aluminum, under the same design and fabrication processes. After characterizing their coherence properties, we found that qubits prepared with tantalum films have the best performance. Since the dry etching process is stable and highly anisotropic, it is much more suitable for fabricating complex scalable quantum circuits, when compared to wet etching. As a result, the current breakthrough indicates that the dry etching process of tantalum film is a promising approach to fabricate medium- or large-scale superconducting quantum circuits with a much longer lifetime, meeting the requirements for building practical quantum computers.

npj Quantum Information (2022)8:3; https://doi.org/10.1038/s41534-021-00510-2

\section{INTRODUCTION}

Quantum computers promise that certain computational tasks such as factorization and quantum simulation can be completed exponentially faster than classical computers. Thanks to the compatibility with semiconductor technology, superconducting quantum computing (SQC) has developed rapidly in recent years and becomes one of the most promising candidates for building practical quantum computers. In the last few years, we witnessed a series of breakthroughs in SQC with transmons, ranging from a realization of quantum supremacy ${ }^{1}$ to quantum chemical simulations ${ }^{2,3}$. Superconducting qubit is an artificial quantum system with a macroscopic size, that is, a dimension of usually $300-500$ micrometers. Compared with other natural candidates of the quantum computer, such as trapped ions, cold atoms, and NV centers, one of the drawbacks of SQC is its relatively short coherence time. As a result, there has been a long battle for improving the coherence time of superconducting qubits, since their birth at the end of the last century. Until now, there is over 5 orders-of-magnitude improvement in the coherence time, and we believe it is far from the end. Although we can reach a lifetime of tens or even hundreds of microseconds for superconducting qubits using the state-of-the-art transmon design and fabrications, it still cannot meet the requirements of a practical SQC system, especially for the error threshold of quantum error correction ${ }^{4,5}$. Longer coherence time provides the potential for higher gate fidelity and larger circuit depth, which are the major limitations for practical quantum computation and quantum simulation.

Transmon or $\mathrm{Xmon}^{6,7}$ is the most widely used qubits in SQC, for their advantages of good coherence, easy coupling, and readout, etc. Their design principles are fundamentally the same, except that one of the capacitor pads of Xmon is grounded. When compared to other kinds of superconducting qubits, such as fluxonium ${ }^{8}$ and $\mathrm{C}$-shunt flux qubit ${ }^{9,10}$, transmon has a much simpler structure, with a single or tunable Josephson junction shunted with a large capacitor. The commonly used junctions in superconducting qubits are $\mathrm{Al}-\mathrm{AlO}_{x}-\mathrm{Al}$ trilayer tunnel junctions fabricated by the double-angle evaporation technique. The shunted capacitors for transmons are usually formed by a coplanar capacitor. To control and readout the qubit, one can design the structures to capacitively or inductively couple the transmons to other circuit elements, including microwave and flux drive lines, readout resonators, and couplers.

A series of candidate superconductors, including elementary metals such as niobium ${ }^{11,12}$, aluminum ${ }^{13}$, tantalum ${ }^{14}$, compounds such as $\mathrm{TiN}^{15}, \mathrm{NbN}^{16}, \mathrm{NbTiN}^{17}$, and granular aluminum ${ }^{18}$, have been explored as the materials for building superconducting quantum circuits. After a lot of practice, it is converged into two popular elementary superconductors, aluminum, and niobium, because of their mature fabrication and stable superconducting properties. However, exploring other materials for improving superconducting quantum circuits remains a key task. In 2020, a Princeton group reported a device using tantalum films in BCC alpha-phase, and the new material platform can greatly improve the coherence time of transmon qubits ${ }^{14}$. In their work, the coherence times exceeded a breakthrough value of $0.3 \mathrm{~ms}$. They used both dry etching and wet etching processes to fabricate the qubit circuits, and their results showed that qubits fabricated by wet etching were better than those by dry etching. However, as dry etching technology has a lot of advantages when compared to wet etching, including high anisotropy, the capability of automation, reduced material consumption, good industrial hygiene, etc., it is now widely used in the semiconductor industry, and is also promising for fabricating large-scale solid-state quantum circuits. As a result, it is necessary to explore a robust dry etching process for fabricating transmon qubits with a long lifetime.

In this work, we developed and optimized the dry etching process for Ta films. We fabricated a series of transmon qubits with a very long coherence time using such a dry etching process. The best coherence time reached $503 \mu \mathrm{s}$. As a comparison, we also prepared transmon samples with $\mathrm{Nb}$ and $\mathrm{Al}$, using the

${ }^{1}$ Beijing Academy of Quantum Information Sciences, 100193 Beijing, China. ${ }^{凶}$ email: xuegm@baqis.ac.cn; jinyr@baqis.ac.cn; hfyu@baqis.ac.cn 




same design. All samples are prepared using the same fabrication processes except that the materials of qubit pads are different. After characterizing the coherence properties of the transmons with different materials, we found that tantalum stands out systematically. We further analyzed the source of decoherence, and recognized the material interfaces, including metal-air (MA), metal-substrate (MS), and metal-metal (MM), as important loss channels of the qubits. As dry etching is a powerful technology suitable for the fabrication of large-scale quantum circuits, the current breakthrough indicates that Ta films with a dry etching process are promising for the fabrication of extremely long-lived multi-qubits for large-scale quantum computers.

\section{RESULTS}

\section{Characterization of the coherence properties of different qubits}

We prepared eight chips of different batches and put them into a low-noise dilution refrigerator for measurement, and detailed wiring setups can be found in Supplementary Methods. The specific coherence properties are listed in Table 1.

It can be seen from Table 1 that the Ta transmons outperform the Al and $\mathrm{Nb}$ ones systematically. $Q_{3}$ of sample Ta-4, $Q_{1}$ of Ta-3, and $Q_{1}$ of Ta-2 were measured repeatedly for a longtime interval, as shown in Fig. 1. For $Q_{3}$ of sample Ta-4, the average value of qubit relaxation time $T_{1}$ is $401 \mu \mathrm{s}$, and the best value reaches $503 \mu \mathrm{s}$. For $Q_{1}$ of Ta-3, the average value of $T_{1}$ equals $356 \mu \mathrm{s}$, and the best value is $383 \mu \mathrm{s}$. For $Q_{1}$ of Ta-2, the average value of $T_{1}$ equals $359 \mu \mathrm{s}$ with the best value of $431 \mu \mathrm{s}$.

Although the fabrication processes of chips Ta-2, Ta-3, and Ta-4 are the same, their relaxation time $T_{1}$ could vary from $102.7 \mu$ s to $476 \mu \mathrm{s}$, and $T_{1}$ values also fluctuate on the same chip. We speculate that the variation of $T_{1}$ from chip to chip is most probably due to the fluctuation of the fabrication process, while the variation of $T_{1}$ among qubits on the same chip mainly arises from two-level systems ${ }^{19}$ and quasiparticles ${ }^{20}$. It is also noticed that the $T_{1}$ values of the Ta- 1 chip are lower than those of other Ta chips. This might be due to the fact that the Ta-1 chip was not dipped in Piranha while the others were. Piranha can strongly oxidize the tantalum surface apart from removing organic impurities. We already know that post-oxidation of aluminum leads to higher quality factor and we assume that post-oxidation of tantalum should have similar effects. However, it is still not clear whether the active post-oxidation or the removal of impurities contribute to a longer lifetime.

We also carried out the Carr-Purcell-Meiboom-Gill (CPMG) echo experiment ${ }^{21}$ for $Q_{1}$ of sample Ta-2. Here, two $\pi$-pulses along the $y$-axis were inserted between two $\pi / 2$ pulses along the $x$-axis, and the CPMG echo obtains a qubit dephasing time $T_{2}{ }^{\text {CPMG2 }}$ of $557 \mu \mathrm{s}$ (see Supplementary Fig. 4).

\section{DISCUSSION}

Next, we analyze the reasons for causing the different relaxation times in different materials. For a fixed frequency transmon qubit, the charge noise and flux noise are suppressed, and dielectric loss from the sapphire substrate was estimated to exceed $10 \mathrm{~ms}^{14}$. We also carefully shielded the qubit during measurement. As we know, the major source of decoherence comes from the two-level system (TLS) defects in the material interface ${ }^{22}$, including metalsubstrate (MS) interface between $\mathrm{Nb}$ ( $\mathrm{Al}$ or $\mathrm{Ta}$ ) films and the substrates, metal-metal (MM) interface between $\mathrm{Nb}(\mathrm{Al}$ or $\mathrm{Ta})$ and Al Junction films, and metal-air (MA) interface between $\mathrm{Nb}$ ( $\mathrm{Al}$ or $\mathrm{Ta}$ ) and air. The sapphire substrates in our devices were carefully handled by chemical cleaning and annealing. Before film deposition, we heated them in a load-lock chamber $\left(200^{\circ} \mathrm{C}\right.$, 2 hours) for degassing. We made chemical cleaning and annealing 

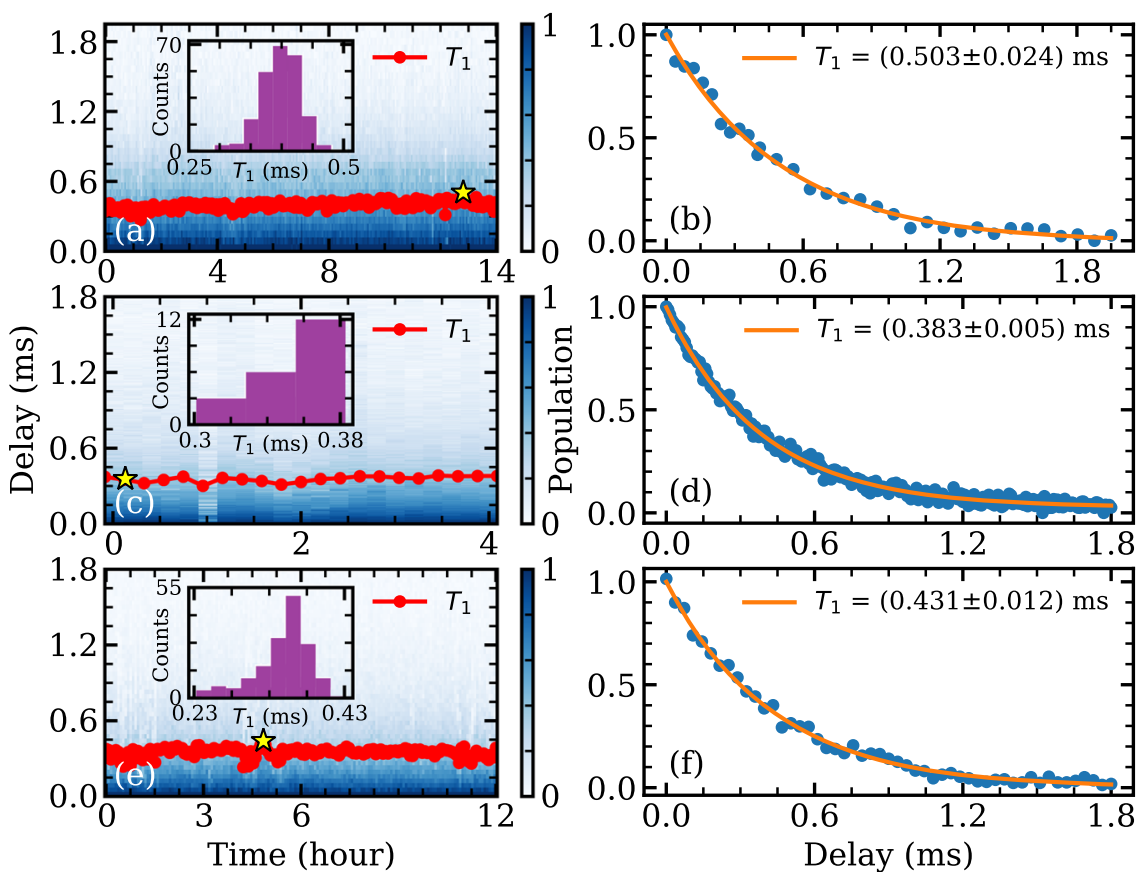

Fig. 1 The relaxation times of three qubits. Panels $\mathbf{a}$, c, e show relaxation times of $Q_{3}$ in Ta- 4 chip, $Q_{1}$ in Ta-3 chip, and $Q_{1}$ in Ta-2 chip measured in 14,4 , and $12 \mathrm{~h}$, respectively. The insets are the corresponding histograms, and the yellow stars indicate the best relaxation time values during each time interval. The orange lines in $\mathbf{b}, \mathbf{d}, \mathbf{f}$ are the fitting curves of the best relaxation measurement results, respectively; the average and best times are $401 \mu \mathrm{s}$ and $503 \mu \mathrm{s}$ for $Q_{3}$ in Ta-4, $356 \mu$ s and $383 \mu \mathrm{s}$ for $Q_{1}$ in Ta-3, and $359 \mu \mathrm{s}$ and $431 \mu \mathrm{s}$ for $Q_{1}$ in Ta-2, respectively.

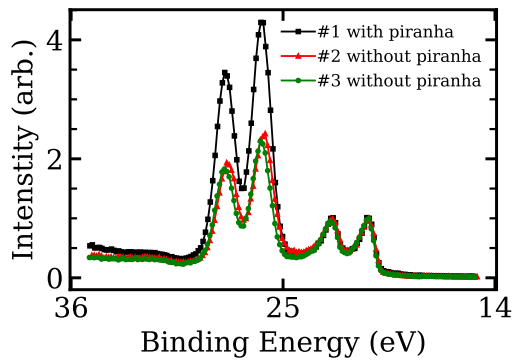

Fig. 2 The XPS spectra of three Ta samples. Two peaks at low binding energy belong to $4 f^{7 / 2}$ and $4 f^{5 / 2}$ orbitals of tantalum metal, and two peaks at higher energy correspond to the same orbitals of $\mathrm{Ta}_{2} \mathrm{O}_{5}$. The black line indicates that the sample has been dipped into piranha solution for $20 \mathrm{~min}$ with a temperature over $70^{\circ} \mathrm{C}$, and shows thicker tantalum oxide than others that are not dipped into piranha solution.

to reduce the loss from the MS interface. The same is true for the MM interface: before fabrication of the junctions, $\mathrm{Nb}$ (Al or $\mathrm{Ta}$ ) was cleaned with a radio frequency ion source and over-etched for 30 seconds to ensure that the oxide layer was removed completely. Although the interface properties between $\mathrm{Nb}-\mathrm{Al}$, $\mathrm{Al}-\mathrm{Al}$, and Ta-Al are different, there are no contaminants introduced in this process. After using the steps above, we have optimized all interfaces except MA. As the metal is exposed to the atmosphere during fabrication and packaging, various components in the air can form compounds (mainly oxides) with the metal. These oxides contain various defects that may couple with the qubits and cause decoherence. The defects from the surface oxides have been carefully studied by many research groups. Niobium oxides have three components: $\mathrm{NbO}, \mathrm{NbO}_{2}$, and $\mathrm{Nb}_{2} \mathrm{O}_{5}{ }^{23,24}$. Verjauw et al. ${ }^{25}$ found that if the niobium oxides were removed by hydrofluoric (HF) acid etching, the internal quality factor of the resonator could reach up to 7 million at a

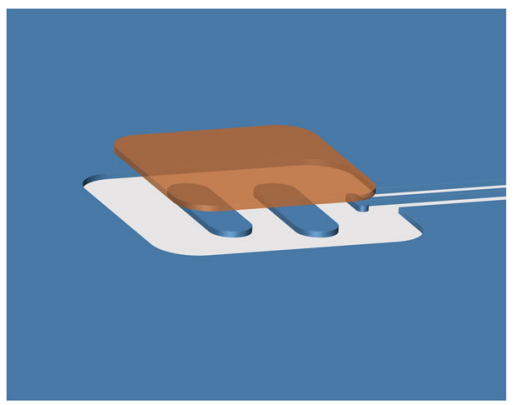

Fig. 3 A schematic picture of a transmon prepared by the flip-chip process. It consists of two substrates with patterned tantalum films. The two substrates are packaged face-to-face with indium bumps using the flip-chip technology. The Josephson junction is located between the two pads (blue color) on the bottom substrate (Josephson junction is not shown in this picture), and the Ta film pattern (orange color) on the upper substrate is located directly above the two pads of the bottom substrate. The purpose of this is to increase the SPR of the MA interface because there is an electric field that is localized between the upper and lower substrates.

single-photon power level, while that of the reference sample with intact native oxides is only 1 million. This provides solid evidence that the TLS defects located in the MA interfaces play an important role in its loss tangent. Premkumar et al. also systematically compared the effect of different $\mathrm{Nb}$ oxides with $T_{1}^{25,26}$. For aluminum films, the surface oxides were formed by oxidation in load lock after evaporation. The resulting surface aluminum oxides were reported to have an O/Al ratio of $0.9-1.1^{27}$. Thus it may contain $\mathrm{O}-\mathrm{H}$ and $\mathrm{O}-\mathrm{O}$ bonds and hydrogenated $\mathrm{Al}$ vacancies, which can contribute to TLS loss ${ }^{28}$. As to tantalum, its oxide has only one component, $\mathrm{Ta}_{2} \mathrm{O}_{5}$. As shown in Fig. 2, we have measured three samples (one is cleaned in piranha solution) using 


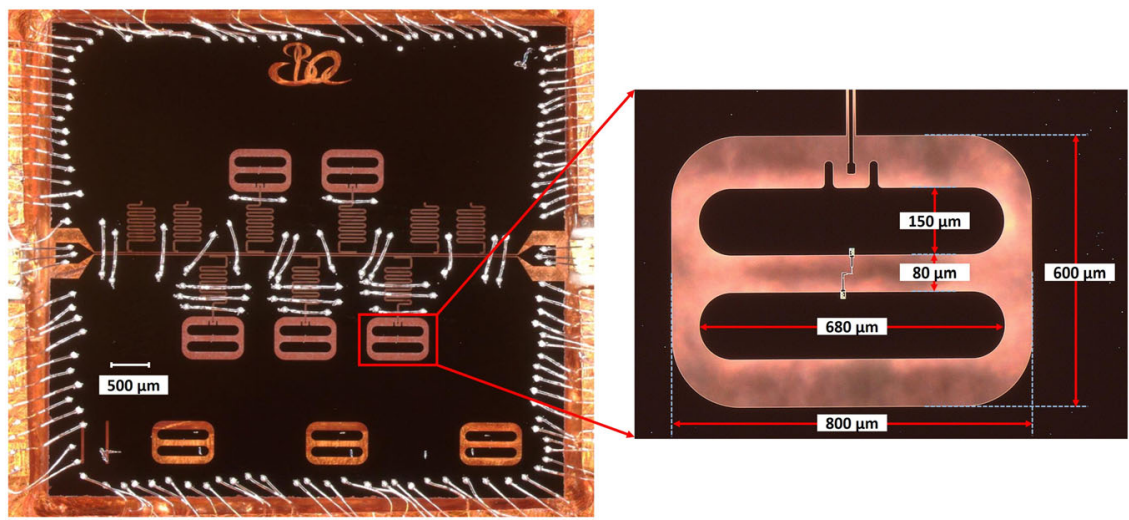

Fig. 4 An optical micrograph of a packaged transmon sample with Ta film. The size of the chip is $7 \mathrm{~mm}$. It contains five independent transmons, four independent quarter-wave resonators for measuring the intrinsic $Q$ factor of the resonator, and three transmons for the resistance test. Purcell limit of transmon design is over 2 milliseconds. The coupling strength between the readout resonator and transmon is $50 \mathrm{MHz} \times 2 \pi$ (experimental values are close to the design values). The dimensions in the enlarged photo are design values.
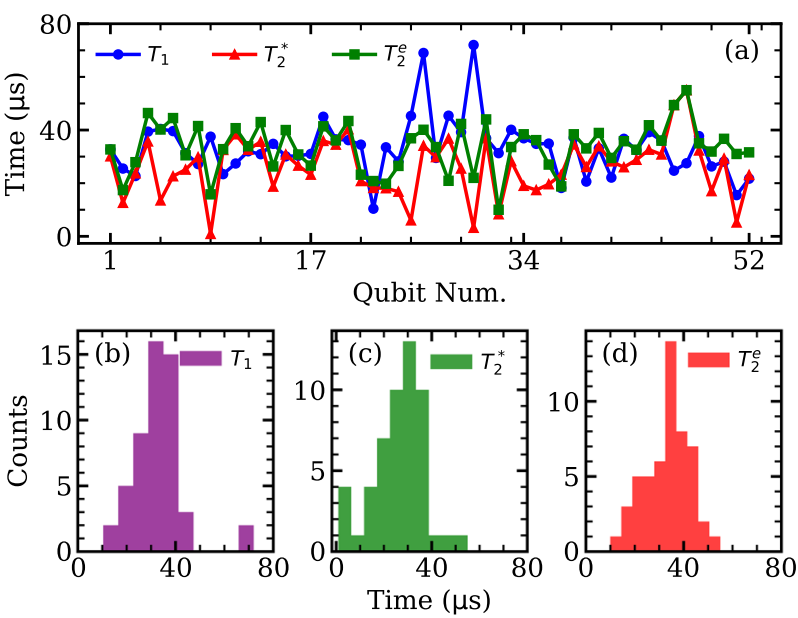

Fig. $5 T_{1}, T_{2}^{*}$, and $T_{2}{ }^{\mathrm{e}}$ of each qubit of a multi-qubit chip with Ta film. a $T_{1}$ (blue), $T_{2}{ }^{*}$ (red), and $T_{2}{ }^{\mathrm{e}}$ (green) characterization results of a 56-qubits chip fabricated with the same processes described here. (b-d) are histograms of the $T_{1}, T_{2}^{*}$, and $T_{2}{ }^{\mathrm{e}}$ of all the qubits in the chip, respectively.

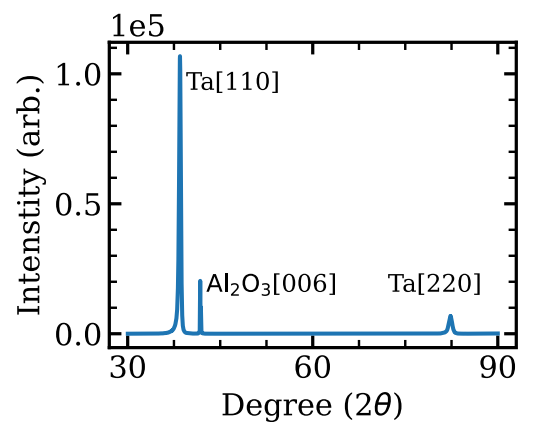

Fig. 6 XRD spectrum of a Ta film on sapphire. Two clear peaks correspond to [110] and [220] of the alpha phase of $\mathrm{Ta}$, and the peak in the middle corresponds to the sapphire substrate we used.

XPS to obtain chemical elements of Ta film surface. The two peaks at low binding energy belong to $4 f^{7 / 2}$ and $4 f^{5 / 2}$ orbitals of tantalum metal, and the two peaks at higher binding energy correspond to the same orbitals of $\mathrm{Ta}_{2} \mathrm{O}_{5}{ }^{10}$. We do not see any other chemical components, which indicates that $\mathrm{Ta}_{2} \mathrm{O}_{5}$ is the only surface oxide of Ta films. From our results, it can be seen that without surface treatment, the results of Ta transmons are better than those of $\mathrm{Nb}$ and $\mathrm{Al}$ ones. However, if one can manage to remove the surface oxide layers of the Ta films or the $\mathrm{Nb}$ films (because the cleaning process is not selective, the oxide layer on the surface of Al cannot be removed without damaging the junctions) and keep the surface clean during the measurements in the fridges, the coherence times are expected to be further improved. This may be accomplished by using, for example, HF vapor to remove oxides and vacuum packaging to keep the surfaces from being recontaminated. Further experiments are needed to verify this.

We also made a comparison with a sample specially designed with a high MA surface participation ratio (SPR), as shown in Fig. 3. By using flip-chip technology, we introduced an additional Ta pad over the Ta transmon with a height of about 5 micrometers (the total capacitance of this type of qubits is controlled to be equal to that used in Table 1, with the charging energy of $E_{\mathrm{c}} / 2 \pi \sim 260 \mathrm{MHz}$ ). The purpose of this design is to deliberately increase the SPR of MA. The measured $T_{1}$ and $T_{2}{ }^{*}$ are $50 \mu \mathrm{s}$ and $75 \mu \mathrm{s}$, respectively, which are much lower than the former design of Fig. 4. Simulations of SPR of relevant interfaces are shown in Supplementary Discussion, supporting our conjecture that MA plays an important role in the decoherence of our qubits. A more detailed data analysis of this type of qubits is presented in reference ${ }^{29}$.

Practical SQC requires large-scale integration of the qubits. As a result, we also designed and fabricated quantum circuits with 56 qubits and 55 couplers, using the same processes for our longlived Ta transmons. The Purcell limit of our design exceeds $1 \mathrm{~ms}$. Figure 5 shows the $T_{1}, T_{2}{ }^{*}$, and $T_{2}{ }^{\mathrm{e}}$ of each fixed-frequency qubit in a chip (coherence times of the couplers are not plotted), all of which are much lower than those listed in Table 1 . We partly attribute it to the environmental noise of the chip. For our single qubit chip, the cables for control and measurement were deeply attenuated and filtered; however, the same situation cannot be achieved in a multi-qubit chip, since we need to control and bias the qubits quickly enough. This causes high-frequency noises to the qubits through cables and results in decoherence. Therefore, the environmental noise of the measurement system should be carefully suppressed in order to improve the coherence time of large-scale quantum circuits. This is a challenging issue at the current stage.

In this work, we used the dry etching process for Ta film to prepare single-qubit samples, the best lifetime of which exceeds $500 \mu \mathrm{s}$. These results show that dry etching can be adopted in the subsequent preparation of tantalum qubits, which provides a powerful method for large-scale fabrication. We also compared the coherence time of the qubits with the same design prepared by $\mathrm{Nb}$ and $\mathrm{Al}$. We found that the performance of $\mathrm{Ta}$ is the best. In 
Table 2. The etching parameters for the two etching machines.

\begin{tabular}{llllll}
\hline Etching machine & Etching gas mixture & Pressure (mTorr) & Plasma power (Watts) & Bias power (Watts) & Etching time (seconds) \\
\hline ICP & $\mathrm{SF}_{6}: \mathrm{CHF}_{3}=4: 1$ & 4 & 220 & 50 & $180($ for $150 \mathrm{~nm})$ \\
RIE & $\mathrm{CF}_{4}$ & 15 & 100 & - & 180 (for $150 \mathrm{~nm})$ \\
\hline
\end{tabular}
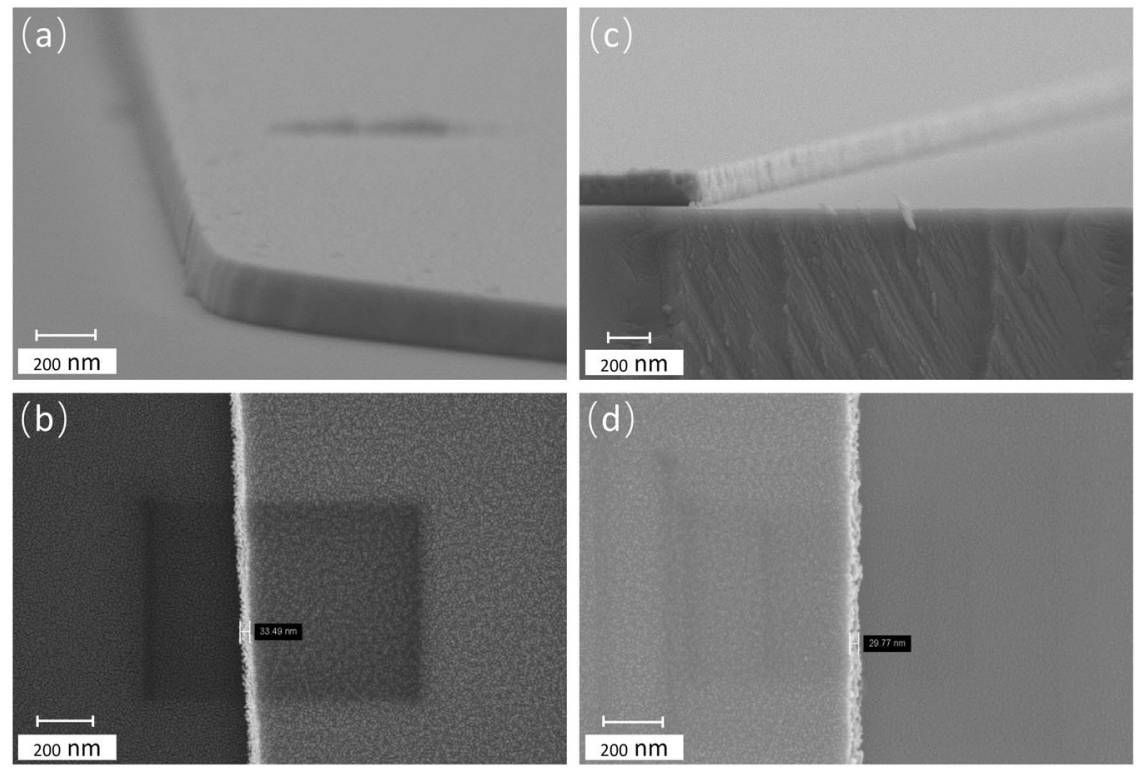

Fig. 7 Four SEM images of Ta film after dry etching. Panels $\mathbf{a}$ and $\mathbf{b}$ show different angles of the same sample, which was etched in RIE etcher using $\mathrm{CF}_{4}$ gas. Same for $\mathbf{c}$ and $\mathbf{d}$, but $\mathrm{SF}_{6}: \mathrm{CHF}_{3}$ mixed gases were used in the ICP etcher. The measured widths of the film edge of both samples are around $30 \mathrm{~nm}$. In $\mathbf{c}$, the horizontal edge is the interface between the substrate and the above metal and air, and the thinner oblique edge is for the tantalum film.

addition, we deliberately designed a flip-chip transmon with a high SPR of MA. The comparison of $T_{1}$ and $T_{2}{ }^{\mathrm{e}}$ between the flipchip transmons and conventional ones showed that dielectric loss from interfaces is still the main source for the decoherence of the qubits. The current breakthrough in coherence times indicates that the dry etching process for Ta film can be used to fabricate transmon qubits with extremely long lifetimes, and our method greatly promotes the performance of superconducting qubits. Furthermore, the dry etching technique used in this work also provides a powerful way for fabricating large-scale quantum circuits. With the continuous improvement and optimization of the material interfaces, we believe that transmon qubits with a coherence time of milliseconds or more could be achieved soon.

\section{METHODS}

\section{Sample design and fabrication}

In order to obtain a longer coherence time, we optimized the sample design as follows. First, a single-junction design of fixed-frequency transmon was adopted, and this design greatly reduces the influence of flux noise. Second, to suppress noise from the environment, the circuit is minimized to only two necessary electrodes for the feedline, without delicated control lines for individual qubits. The circuit includes five transmons, each of which is dispersively coupled to a readout resonator and then coupled to a transmission line serving as the feedline. As a result, the microwave drive pulses and readout pulses are both inputted from one port of the transmission line. Finally, each qubit has a shunted capacitor with an enlarged pad area to decrease the electric field density and reduce the impact of surface loss, similar to the design of Princeton and IBM groups ${ }^{11}$. An optical micrograph of one sample is shown in Fig. 4.

With the above design, $\mathrm{Nb}, \mathrm{Al}$, and Ta films were used to prepare the base metallization layer of the transmon samples. The preparation process is as follows. A superconducting film with a thickness of about $120 \mathrm{~nm}$ was deposited on a sapphire substrate. The substrate was chemically cleaned and annealed up to $1100^{\circ} \mathrm{C}$ prior to the deposition. The $\mathrm{Nb}$ and Ta films were prepared by $\mathrm{dc}$ magnetron sputtering, and the Al films were prepared by electron beam evaporation. Transmon pads, readout resonators, and the transmission line were patterned by ultraviolet (UV) lithography (DWL66 + from Heidelberg instruments Mikrotechnik GmbH) with a single layer S1813 resist. After development, we used inductively coupled plasma (ICP) or reactive ion etching (RIE) systems to remove the unwanted films. Then, Dolan bridges were prepared by electron beam lithography (EBL) with PMMA A4/LOR10B double-layer photoresists. Al$\mathrm{AlO}_{x}-\mathrm{Al}$ Josephson junctions were prepared using a four-chamber E-beam evaporator (JEB-4 from AdNaNoTek Corp.). Before the preparation of Al junctions, a radio frequency ion source was used to clean the oxides on the surface of the ground metal ( $\mathrm{Nb}, \mathrm{Al}$, or $\mathrm{Ta}$ ) to achieve a superconducting connection. After wafer dicing, a liftoff process in N-Methylpyrrolidone (NMP) solution was performed to remove the photoresists and unwanted aluminum. Finally, the chip was wire-bonded into a copper sample box.

We optimized the deposition conditions for $\mathrm{Nb}$ and Ta films, especially for Ta films of BCC alpha-phase, including sputtering pressure, deposition speed, working distance between the target and the substrate, substrate temperature, etc. Finally, we obtained $\mathrm{Nb}$ films with a typical residual resistance ratio (RRR) $300 \mathrm{~K} / 10 \mathrm{~K}$ of 4.9 and a critical temperature $\left(T_{\mathrm{c}}\right)$ of $9.1 \mathrm{~K}$, while for Ta films RRR $=4.5$ and $T_{\mathrm{c}}=4.2 \mathrm{~K}$. Figure 6 shows the XRD result of a Ta film of alpha-phase, and this result shows that Ta films are pure alpha-phase without discernable beta-phase component.

When exploring the dry etching process for Ta films, we used two etching machines, one is an ICP etcher (PlasmaPro 100 Cobra from Oxford Instruments), which has two radio frequency sources, and the other is an RIE etcher (200NL from Samco). After many rounds of process optimization, we finally obtained two sets of optimized etching parameters, shown in Table 2. Figure 7 shows the scanning electron microscope (SEM) photos of different edges prepared by both etchers. From the SEM photos, we found that the etching effects of the two etchers are almost the same, with 
smooth and clean edges. As a result, they are both suitable for fabricating circuits. The Ta transmons in this paper were prepared by the RIE etcher with $\mathrm{CF}_{4}$ gas.

\section{DATA AVAILABILITY}

All data needed to evaluate the conclusions in the paper are present in the main text and/or the Supplementary Information. Additional data related to this paper may be requested from the authors.

Received: 7 July 2021; Accepted: 28 November 2021; Published online: 13 January 2022

\section{REFERENCES}

1. Arute, F. et al. Quantum supremacy using a programmable superconducting processor. Nature 574, 505-510 (2019).

2. Arute, F. et al. Hartree-Fock on a superconducting qubit quantum computer. Science 369, 1084-1089 (2020).

3. Kandala, A. et al. Hardware-efficient variational quantum eigensolver for small molecules and quantum magnets. Nature 549, 242-246 (2017).

4. Kjaergaard, M. et al. Superconducting qubits: current state of play. Annu. Rev. Condens. Matter Phys. 11, 369-395 (2020).

5. Barends, R. et al. Superconducting quantum circuits at the surface code threshold for fault tolerance. Nature 508, 500-503 (2014).

6. Koch, J. et al. Charge-insensitive qubit design derived from the Cooper pair box. Phys. Rev. A 76, 042319 (2007).

7. Barends, R. et al. Coherent Josephson qubit suitable for scalable quantum integrated circuits. Phys. Rev. Lett. 111, 080502 (2013).

8. Manucharyan, V. E., Koch, J., Glazman, L. I. \& Devoret, M. H. Fluxonium: single Cooper-pair circuit free of charge offsets. Science 326, 113-116 (2009).

9. You, J. Q., Hu, X., Ashhab, S. \& Nori, F. Low-decoherence flux qubit. Phys. Rev. B 75, 140515(R) (2007).

10. Yan, F. et al. The flux qubit revisited to enhance coherence and reproducibility. Nat. Commun. 7, 12964 (2016).

11. Gambetta, J. M. et al. Investigating surface loss effects in superconducting transmon qubits. IEEE Trans. Appl. Supercond. 27, 1700205 (2017).

12. Nersisyan, A. et al. Manufacturing low dissipation superconducting quantum processors. in 2019 IEEE International Electron Devices Meeting (IEDM). (IEEE, 2019).

13. Paik, $\mathrm{H}$. et al. Observation of high coherence in Josephson junction qubits measured in a three-dimensional circuit QED architecture. Phys. Rev. Lett. 107, 240501 (2011)

14. Place, A. P. M. et al. New material platform for superconducting transmon qubits with coherence times exceeding 0.3 milliseconds. Nat. Commun. 12, 1779 (2021).

15. Chang, J. et al. Improved superconducting qubit coherence using titanium nitride. Appl. Phys. Lett. 103, 012602 (2013).

16. Kim, S. et al. Enhanced coherence of all-nitride superconducting qubits epitaxially grown on silicon substrate. Commun. Mater. 2, 98 (2021).

17. Samkharadze, N. et al. High-kinetic-inductance superconducting nanowire resonators for circuit QED in a magnetic field. Phys. Rev. Appl. 5, 044004 (2016).

18. Winkel, P. et al. Implementation of a transmon qubit using superconducting granular aluminum. Phys. Rev. X 10, 031032 (2020).

19. Klimov, P. V. et al. Fluctuations of energy-relaxation times in superconducting qubits. Phys. Rev. Lett. 121, 090502 (2018).

20. Vepsäläinen, A. P. et al. Impact of ionizing radiation on superconducting qubit coherence. Nature 584, 551-556 (2020).

21. Bylander, J. et al. Noise spectroscopy through dynamical decoupling with a superconducting flux qubit. Nat. Phys. 7, 565-570 (2011).

22. Dial, O. et al. Bulk and surface loss in superconducting transmon qubits. Supercond. Sci. Technol. 29, 044001 (2016).

23. Lindau, I. \& Spicer, W. E. Oxidation of $\mathrm{Nb}$ as studied by the UV-photoemission technique. J. Appl. Phys. 45, 3720 (1974).
24. Jia, X. Q. et al. High performance ultra-thin niobium films for superconducting hot-electron devices. IEEE Trans. Appl. Supercond. 23, 2300704 (2012).

25. Verjauw, J. et al. Investigation of microwave loss induced by oxide regrowth in high-Q Nb resonators. Phys. Rev. Appl. 16, 014018 (2021).

26. Premkumar, A. et al. Microscopic relaxation channels in materials for superconducting qubits. Commun. Mater. 2, 72 (2021).

27. Tan, E., Mather, P. G., Perrella, A. C., Read, J. C. \& Buhrman, R. A. Oxygen stoichiometry and instability in aluminum oxide tunnel barrier layers. Phys. Rev. B 71, 161401(R) (2005).

28. Müller, C., Cole, J. H. \& Lisenfeld, J. Towards understanding two-level-systems in amorphous solids: insights from quantum circuits. Rep. Prog. Phys. 82, 124501 (2019).

29. Li, X. et al. Vacuum-gap transmon qubits realized using flip-chip technology. Appl. Phys. Lett. 119, 184003 (2021).

\section{ACKNOWLEDGEMENTS}

We thank Dr. Rui Wu for her fruitful suggestions on Ta film growth. This work was supported by the NSF of Beijing (Grant No. Z190012), the NSFC of China (Grant No. 11890704, 12004042), National Key Research and Development Program of China (Grant No. 2016YFA0301800), and the Key-Area Research and Development Program of Guang Dong Province (Grant No. 2018B030326001).

\section{AUTHOR CONTRIBUTIONS}

The project was conceived by H.Y., Y.J., and G.X. The devices are designed by G.X. and Y.Z. The devices are fabricated by C.W., Z.M., T.S., and G.X. The measurements were performed by X.L., H.X., Z.L., J.W., and Z.Y. The experimental data were analyzed by X.L., H.X., Z.L., J.W., and Z.Y. H.Y. wrote the manuscript together with Y.J. and G.X. All authors discussed the results and the manuscript.

\section{COMPETING INTERESTS}

The authors declare no competing interests.

\section{ADDITIONAL INFORMATION}

Supplementary information The online version contains supplementary material available at https://doi.org/10.1038/s41534-021-00510-2.

Correspondence and requests for materials should be addressed to Guangming Xue, Yirong Jin or Haifeng Yu.

Reprints and permission information is available at http://www.nature.com/ reprints

Publisher's note Springer Nature remains neutral with regard to jurisdictional claims in published maps and institutional affiliations.

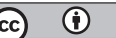

Open Access This article is licensed under a Creative Commons Attribution 4.0 International License, which permits use, sharing, adaptation, distribution and reproduction in any medium or format, as long as you give appropriate credit to the original author(s) and the source, provide a link to the Creative Commons license, and indicate if changes were made. The images or other third party material in this article are included in the article's Creative Commons license, unless indicated otherwise in a credit line to the material. If material is not included in the article's Creative Commons license and your intended use is not permitted by statutory regulation or exceeds the permitted use, you will need to obtain permission directly from the copyright holder. To view a copy of this license, visit http://creativecommons. org/licenses/by/4.0/.

(c) The Author(s) 2022 